\title{
A comparison of predictors and intensity of acute postsurgical pain in patients undergoing total hip and knee arthroplasty
}

This article was published in the following Dove Press journal:

Journal of Pain Research

9 May 2017

Number of times this article has been viewed

\author{
Patrícia R Pinto ${ }^{1,2}$ \\ Teresa Mclntyre ${ }^{3}$ \\ Vera Araújo-Soares ${ }^{4}$ \\ Patrício Costa ${ }^{1,2,5}$ \\ Ramón Ferrero 6 \\ Armando Almeida ${ }^{1,2}$ \\ 'Life and Health Sciences Research \\ Institute (ICVS), School of Medicine, \\ University of Minho, Braga, 'ICVS/3B's \\ - PT Government Associate \\ Laboratory, Braga/Guimarães, \\ Portugal; ' ${ }^{3}$ Department of Psychology, \\ Houston Baptist University, Houston, \\ TX, USA; ${ }^{4}$ Institute of Health and \\ Society, Faculty of Medical Sciences, \\ Newcastle University, Newcastle upon \\ Tyne, UK; ${ }^{5}$ Faculty of Psychology and \\ Education Sciences, University of \\ Porto, Porto, Portugal; ${ }^{6}$ Orthopedics \\ Unit, Alto Ave Hospital Center, \\ Guimarães, Portugal
}

Background: Acute pain is an expected result after surgery. Nevertheless, when not appropriately controlled, acute pain has a very negative impact on individual clinical outcomes, impairing healing and recovery, and has clear consequences on health care system costs. Augmenting knowledge on predictors and potentially modifiable determinants of acute postsurgical pain can facilitate early identification of and intervention in patients at risk. However, only a few studies have examined and compared acute pain after total hip arthroplasty (THA) and total knee arthroplasty (TKA). The aim of this study was to compare THA and TKA in acute postsurgical pain intensity and its predictors.

Methods: A consecutive sample of 124 patients with osteoarthritis (64 undergoing THA and 60 TKA) was assessed 24 hours before (T1) and 48 hours after (T2) surgery. Demographic, clinical, and psychological factors were assessed at $\mathrm{T} 1$, and acute postsurgical pain experience was examined at T2. Additionally, the same hierarchical regression analysis was performed separately for each arthroplasty type.

Results: TKA patients reported higher levels of acute postsurgical pain compared with THA ( $t=8.490, p=0.004, d=0.527,95 \%$ confidence interval, $0.196-0.878$ ). In the final THA predictive model, presurgical pain was the only variable approaching significant results $(t[57]=1.746$, $\beta=0.254, p=0.086$ ). In the final TKA predictive model, optimism was the only predictor of pain $(t[51]=-2.518, \beta=-0.339, p=0.015)$, with emotional representation $(t[51]=1.895, \beta=0.254$, $p=0.064$ ) presenting a trend toward significance.

Conclusion: The current study is the first examining THA and TKA differences on acute postsurgical pain intensity and its predictors using a multivariate approach. Results from this study could prove useful for the design of distinct interventions targeting acute postsurgical pain management depending on whether the site of arthroplasty is the hip or the knee. Finally, the current results also support the argument that these two surgeries, at least with regard to acute pain, should be approached separately.

Keywords: acute post-surgical pain, total knee arthroplasty, total hip arthroplasty, psychological factors, multivariate analyses, presurgical psychological intervention

\section{Introduction}

Osteoarthritis (OA) of major joints is a progressive disabling disease and a major cause of chronic pain, which might result in patients undergoing total hip arthroplasty (THA) or total knee arthroplasty (TKA). ${ }^{1}$ These are high-cost procedures that are not always successful. ${ }^{2}$

Several studies have examined the predictors of medium-to-long-term outcomes of these type of surgeries, including chronic pain..$^{2-14}$ Yet, there are not so many studies examining acute postsurgical pain predictors, within a multivariate approach.
Correspondence: Patrícia R Pinto Institute (ICVS), School of Medicine, Campus de Gualtar, University of Minho, 47 I0-057 Braga, Portugal

Tel +35I 253604926

Fax +35I 253604809

Email patipinto@med.uminho.pt 
Nonetheless, acute pain is a crucial issue, given that unless properly managed, it creates needless suffering, delays healing processes, puts patients at risk of increased postsurgical morbidity and mortality, and increases hospital stay and costs of care. ${ }^{15-17}$ Overall, it may have detrimental effects in both physiological and psychological domains ${ }^{18,19}$ and might lead to the development of chronic pain. ${ }^{16}$ Therefore, understanding how to prevent acute postsurgical pain early in the recovery process can support better and more efficient recovery from surgery. ${ }^{17,20,21}$ Augmenting knowledge on predictors and potentially modifiable determinants of acute postsurgical pain can facilitate early identification of and intervention in patients at risk.

Indeed, several studies focused on the study of acute pain predictors after different types of surgery. ${ }^{20,22-28}$ Concerning prediction of acute pain after THA and TKA, we are aware of two studies wherein TKA and THA were approached jointly, ${ }^{29,30}$ and others wherein only TKA was considered, ${ }^{31-40}$ with only two studies ${ }^{41,42}$ focusing specifically on THA, thus leaving a considerable gap of knowledge in this field.

In addition, there is one key question related to the comparison of pain experience after THA and TKA. Regarding chronic postsurgical pain, differences between these two approaches are well established, with worst results for TKA, ${ }^{5-8}$ and pointing to the more established predictive role of psychological variables on pain after TKA than after THA. ${ }^{9}$

Concerning acute postsurgical pain, empirical knowledge reveals higher acute pain levels after TKA when compared to THA, although the scarce published literature on this ${ }^{43-45}$ is not enough to uphold any definitive conclusion. The abovementioned studies had a very small sample of patients, presenting sampling power issues. Moreover, Aarons et $\mathrm{al}^{43}$ and Wylde et $\mathrm{al}^{45}$ did not use a multivariate approach to analyze the differences on acute postsurgical pain with surgery type. Pang et $\mathrm{al}^{44}$ relied on morphine consumption, not directly comparing pain intensity scores as reported by patients. However, given the idiosyncrasy of pain perception, using participant scores would have also been crucial. Finally, none of the abovementioned studies sought specific predictors of acute pain.

Research is needed to draw definitive conclusions regarding heightened acute pain in TKA in comparison to THA, and most importantly to distinguish acute pain predictors according to arthroplasty type. This study aims to compare acute postsurgical pain intensity, and its potential predictors, between two types of major joint arthroplasties: THA and TKA.

\section{Methods}

The research was conducted according to the Declaration of Helsinki and ethical approval was granted by the Hospital Research Ethics Committee of Alto Ave Hospital Center in Portugal. All participants were informed about the study and then read and signed the informed consent form.

\section{Participants and procedures}

This study is a post hoc analysis of a prospective cohort single study investigating acute pain and persistent postsurgical pain prevalence among major joint arthroplasties. ${ }^{6,7,30}$ For the purposes of this study, all patients assessed 24 hours before (T1) and 48 hours after (T2) surgery were included, resulting in a consecutive sample of 124 patients with OA (64 THA [38 women, 26 men] and 60 undergoing TKA [45 women, 15 men]). Inclusion criteria were being 18-80 years old, being able to understand written information (informed consent), having no psychiatric or neurologic pathology (eg, psychosis, dementia, etc) and undergoing primary unilateral THA or TKA for diagnosis of coxarthrosis and gonarthrosis only (OA).

\section{Data collection}

All data were collected via questionnaire, in a face-to-face interview, by a trained psychologist.

\section{Presurgical assessment - predictive measures}

A sociodemographic and clinical data questionnaire was administrated to collect various demographic (eg, age, sex) and clinical data (eg, body mass index, comorbidities, chronic back pain, surgical disease onset). Pain experience was evaluated through the Brief Pain Inventory - Short Form (BPI-SF). ${ }^{46}$

Internal consistency of responses to the Portuguese validated versions of psychological questionnaires was assessed using Cronbach's alpha. ${ }^{47}$ The Hospital Anxiety and Depression Scale ${ }^{48}$ was used to measure anxiety $(\alpha=0.79)$ and depression $(\alpha=0.73)$. Seven scales of the Revised Illness Perception Questionnaire ${ }^{49}$ assessed patient's beliefs about the underlying condition that leads to surgery $(\alpha$ [timeline acute $/$ chronic $]=0.97 ; \alpha$ [timeline cyclical duration $]$ $=0.56 ; \alpha$ [consequences $]=0.46 ; \alpha$ [personal control $]=0.80$; $\alpha$ [treatment control] $=0.87 ; \alpha$ [illness coherence $]=0.87$; $\alpha$ [emotional representation] $=0.90)$. Additionally, the Life Orientation Test-Revised ${ }^{50}$ was employed to evaluate the personality trait optimism $(\alpha=0.96)$. The Surgical Fear Questionnaire $^{51}$ assessed specific surgical fears $(\alpha=0.87)$. A detailed description of these questionnaires can be found elsewhere. . $^{6,24,30}$ 
The "Pain Catastrophizing scale" of the Coping Strategies Questionnaire-Revised Form ${ }^{52}$ evaluated pain catastrophizing. Since there were 14 missing values across subjects in the "pain catastrophizing" scale, we conducted a Bayesian imputation (single output file) $)^{53}$ to generate the latent variable "catastrophizing." Confirmatory factor analysis (CFA) revealed satisfactory fit indexes (comparative fit index [CFI] $=0.982$, root mean square error of approximation [RMSEA] $[p \leq 0.05]=0.106[0.062])$ for this observed latent variable $(\alpha=0.94)$.

\section{Postsurgical assessment - primary outcome measure} Acute pain intensity - patients were asked to rate their average and worst pain level within the first 48 hours after surgery on an 11-point Numerical Rating Scale (NRS). For the purposes of the current analyses, a composite measure was calculated, resulting from the sum and mean of the worst and the average pain level. ${ }^{30,54}$ We believe that this measure is more useful and broader as an outcome, since a combination of measures is acknowledged as a good strategy to diminish error ${ }^{55}$ and increase reliability. ${ }^{56}$

\section{Postsurgical assessment - additional measures}

1. Postsurgical pain frequency: patients were asked to define their pain as constant, intermittent, or brief.

2. Rescue analgesia: it was recorded whether rescue analgesics were provided to patients. All protocols (following specific guidelines normally used in the hospital surgical setting) had indications for the prescription of rescue analgesics beyond the standardized analgesic protocol in the face of moderate-to-severe acute postsurgical pain levels (NRS >3).

\section{Clinical variables}

Clinical data, related to surgery, to anesthesia, and to analgesia, were obtained from medical records. Data concerning the description of the surgical procedure can be obtained elsewhere. ${ }^{6,30}$

\section{Anesthetic technique}

In all patients, the mode of anesthesia was determined by the health care team according to the usual standard anesthetic protocols at the hospital, with no research-related change being introduced. The type of anesthesia in use was classified as: 1) locoregional alone ( $n=82 / 66.1 \%)$, which could be spinal anesthesia or epidural anesthesia, or as 2) locoregional (spinal or epidural anesthesia) plus peripheral nerve blocks $(n=42 / 33.9 \%)$. ASA score (physical status classification of the American Society of Anesthesiologists) was recorded, including cases of ASA class I (9/7.3\%), II (91/73.4\%), and III $(24 / 19.4 \%)$.

\section{Postsurgical care}

Anterior-posterior hip and lateral knee X-rays were taken for THA and TKA, respectively, and reviewed after surgery and before the patient was transferred to the infirmary for continued care. The X-rays were reviewed to ensure that the prosthesis was inserted properly and that alignment was correct.

For both types of surgeries, standardized postsurgical nursing and physical therapy protocols were used for all patients. Patients were mobilized out of bed on the second postsurgical day and had a postoperative anticoagulation protocol using low-molecular-weight heparin. Moreover, patients were given systemic prophylactic antibiotics and a prophylactic anticoagulant to decrease deep venous thrombosis risk after surgery. Compression dressings were removed the day after surgery.

\section{Analgesic protocols}

All patients were assigned to a standardized analgesia protocol. It could be intravenous $(n=31 / 25.0 \%)$, epidural $(n=56 / 45.2 \%)$, or perineural $(n=37 / 29.8 \%)$, followed by oral analgesics on subsequent days.

The standardized intravenous protocol was composed of a continuous intravenous infusion (DIB, delivered infusion baloon) of tramadol (300-600 mg), metamizol (6-8 g), and metoclopramide (60 $\mathrm{mg}$ ). The standardized epidural protocol was a continuous epidural infusion (DIB) with ropivacaine $(0.1 \%)$ and fentanyl $(3 \mu \mathrm{g} / \mathrm{mL})(5 \mathrm{~mL} / \mathrm{h})$. Finally, the standardized perineural protocol included a continuous perineural infusion (DIB) with ropivacaine $(0.2 \%, 5 \mathrm{~mL} / \mathrm{h})$. For the three types of protocols, paracetamol (1 g 6/6 h) and nonsteroidal anti-inflammatory drugs (ketorolac $30 \mathrm{mg}$

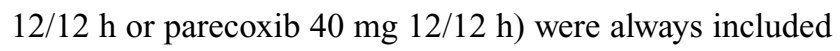
as coadjuvant analgesics. All analgesic regimens included prokinetic treatment were standardized to metoclopramide (10 mg intravenous 8/8 h). All protocols had indications for the prescription of rescue analgesics (ropivacaine and pethidine) beyond the standardized analgesic protocol in cases of moderate-to-severe acute postsurgical pain levels (NRS >3).

\section{Statistical analyses}

Statistical analyses were conducted using the IBM SPSS Statistics (version 22, Armonk, NY, USA) except for the CFA 
and data imputation (Data Collection) that were performed using the IBM SPSS Amos version 22.

Prior to addressing the study questions, we tested whether the underlying assumption of normality was met for all variables. To test this assumption, Kolmogorov-Smirnov tests were used. When the Kolmogorov-Smirnov test was significant, we used the following rules of thumb for ascertaining whether or not to use parametric statistical tests: analyzing the absolute skewness and kurtosis values. If these values were lower than 3.0 and above 8.0, respectively, we followed the indication to proceed with parametric statistics. ${ }^{57}$ No significant violation of normality was observed, with the exception of the variable depression on the THA group (Kurtosis $=11,177$ ).

Descriptive statistics concerning demographic, clinical, and psychological characteristics for each arthroplasty type were computed, and independent $t$-tests (Student's $t$-test, for continuous variables) or $\chi^{2}$ tests ( $\chi^{2}$, for nominal variables) were performed for group comparisons. Statistically significant results were considered for $p<0.05$. To determine the meaningfulness of the differences (practical significance), since statistical significance ( $p$-value) is dependent on group size, the associated effect sizes and $95 \%$ confidence intervals were also computed. Hence, Cohen's $d$ and Pearson's phi ( $\varphi$ ) coefficients were calculated for continuous and nominal variables, respectively. We followed Cohen's ${ }^{58}$ recommendations regarding magnitude of effect sizes. Cohen's $d$ scores above 0.80 were considered a large effect size, between 0.50 and 0.70 a medium effect size, and between 0.20 and 0.40 a small effect size. The interpretation of Pearson's phi $(\varphi)$ coefficient is analogous to the correlation coefficient, expressing the strength of association between two variables.

Pearson correlation coefficients were computed for each arthroplasty type to evaluate the degree of association between acute postsurgical pain and demographic, clinical, and psychological variables to aid in the selection of predictors for the regression model.

One of the aims of this study was to develop and test a similar predictive model separately for acute postsurgical pain intensity after THA and TKA.

The rationale for selecting the predictor variables was the following: 1) inclusion of demographic (age and sex) and clinical (presurgical pain intensity) variables that are acknowledged to influence pain, and more specifically acute postsurgical pain experience; 2) take into account the variables that distinguished the groups presurgically such as disease onset, pain due to other causes, or personal control, for instance, but if the variables distinguished the groups presurgically but did not correlate with the outcome under analysis, then they were not included in the regression model; and 3) select those variables that correlated significantly with the outcome under analysis both in TKA and in THA groups. Hence, optimism and emotional representation were selected since they were the only psychological factors associated significantly with acute postsurgical pain for both groups. The clinical variable (or entitled) other chronic pain states was also selected because it distinguished the groups presurgically, although it only correlated with the outcome in the THA group. The psychological variables depression, pain catastrophizing, and fear were associated with the outcome in the TKA group but were not included in the regression model because they did not distinguish the groups at baseline.

Multiple regression assumptions were verified and multicollinearity was assessed through the variance inflation factor value and the tolerance coefficients, which were established as being below 2 and greater than 0.50 , respectively.

\section{Results \\ Differences between patients undergoing THA and TKA on sociodemographic, clinical, and psychological measures at $\mathrm{TI}$ and $\mathrm{T} 2$}

At $\mathrm{T} 1$, regarding baseline measures, arthroplasty groups did not differ significantly on any sociodemographic characteristic (Table 1), except for age. Patients undergoing TKA were older than those undergoing THA ( $p=0.005, d=0.514)$. Both groups were similar concerning clinical measures, such as body mass index or medical comorbidities, although TKA patients had suffered longer from their surgical disease $(p=0.002, d=0.587$ ). The groups did not differ in terms of presurgical pain intensity and other pain variables (pain interference, other previous chronic pain states, and back pain), but TKA patients revealed more often pain in other joints $(p=0.013, \varphi=0.224)$ (Table 1$)$.

The two arthroplasty groups were similar in terms of their psychological profile (Table 1), with the exception of the illness perception dimensions "personal control" $(p=0.024$, $d=0.411$ ), with TKA patients perceiving more control over the surgical illness (OA) and "illness coherence" $(p=0.019$, $d=0.427$ ), which was also higher, meaning that for them the medical condition leading to surgery makes more sense. Moreover, THA patients revealed higher optimism levels in comparison to TKA patients ( $p=0.017, d=0.436$ ).

Table 1 also shows that in the 48-hour period after surgery (T2), TKA patients exhibited heightened acute postsurgical pain intensity in comparison to those undergoing THA $(p=0.004, d=0.527)$. 
Table I Descriptive statistics on presurgical sociodemographic, clinical, and psychological variables for group differences by arthroplasty type

\begin{tabular}{|c|c|c|c|c|c|c|}
\hline Patient characteristics & $\begin{array}{l}\text { THA } \\
(n=64)\end{array}$ & $\begin{array}{l}\text { TKA } \\
(n=60)\end{array}$ & $\mathbf{t} / \chi^{2}$ & $p$-value & Effect size $^{a}$ & $95 \% \mathrm{Cl}$ \\
\hline \multicolumn{7}{|l|}{ Sociodemographic } \\
\hline Age (years) & $63.3(8.15)$ & $67.2(7.30)$ & -2.860 & 0.005 & 0.514 & {$[0.184-0.864]$} \\
\hline Sex (women) & $38(59.4 \%)$ & $45(75.0 \%)$ & 3.416 & 0.065 & 0.166 & {$[-0.340-0.027]$} \\
\hline Marital status (married) & $50(78.1 \%)$ & $4 \mathrm{I}(68.3 \%)$ & $\mathrm{I} .520$ & 0.218 & 0.111 & {$[-0.082-0.290]$} \\
\hline Residence (rural setting) & $44(68.8 \%)$ & $36(60.0 \%)$ & 1.309 & 0.253 & 0.103 & {$[-0.089-0.287]$} \\
\hline Professional status (retired) & 47 (73.4\%) & $46(76.7 \%)$ & 0.071 & 0.790 & 0.024 & {$[-0.209-0.166]$} \\
\hline \multicolumn{7}{|l|}{ Clinical - general indicators } \\
\hline Disease onset (months) & $78.8(69.9)$ & | 43.2 (| 38.3$)$ & -3.227 & 0.002 & 0.587 & [0.247-0.943] \\
\hline BMI $\left(\mathrm{kg} / \mathrm{m}^{2}\right)$ & $29.0(4.4 I)$ & $30.3(5.21)$ & -1.452 & 0.149 & 0.268 & {$[0.000-0.615]$} \\
\hline Previous surgeries (yes) & $52(82.5 \%)$ & $53(88.3 \%)$ & 0.826 & 0.364 & 0.082 & {$[-0.113-0.247]$} \\
\hline Comorbidities total ${ }^{\mathrm{b}}$ & $1.95(1.28)$ & $2.38(1.12)$ & -1.949 & 0.054 & 0.353 & {$[0.000-0.703]$} \\
\hline \multicolumn{7}{|c|}{ Clinical presurgical pain indicators } \\
\hline NRSc (BPI): Intensity & $7.13(2.21)$ & $6.90(1.87)$ & 0.609 & 0.544 & 0.109 & {$[-0.243-0.462]$} \\
\hline BPI: ${ }^{d}$ Pain Total Interference & $28.3(12.5)$ & $27.7(12.2)$ & 0.283 & 0.778 & 0.051 & {$[-0.302-0.403]$} \\
\hline Other chronic pain states ${ }^{\mathrm{e}}$ (yes) & $37(57.8 \%)$ & $44(75.9 \%)$ & 4.443 & 0.035 & 0.191 & {$[-0.004-0.363]$} \\
\hline Pain in other joints (yes) & $18(21.1 \%)$ & $29(50.0 \%)$ & 6.148 & 0.013 & 0.224 & {$[0.030-0.402]$} \\
\hline Back pain (yes) & $28(43.8 \%)$ & $32(55.2 \%)$ & 1.588 & 0.208 & 0.114 & {$[-0.300-0.079]$} \\
\hline \multicolumn{7}{|l|}{ Psychological measures } \\
\hline HADS: Anxiety & $5.11(4.02)$ & $5.95(4.19)$ & -1.140 & 0.256 & 0.205 & {$[0.000-0.547]$} \\
\hline HADS: Depression & $2.31(3.02)$ & $2.45(3.27)$ & -0.243 & 0.808 & 0.044 & {$[0.000-0.286]$} \\
\hline IPQ-R: Timeline acute/chronic & $8.09(2.67)$ & $8.73(2.98)$ & -1.260 & 0.210 & 0.226 & {$[0.000-0.570]$} \\
\hline IPQ-R: Personal control & $6.25(1.98)$ & $7.18(2.5 \mathrm{I})$ & -2.288 & 0.024 & 0.411 & {$[0.078-0.760]$} \\
\hline IPQ-R: Treatment control & $11.9(1.17)$ & $12.0(1.10)$ & -0.372 & 0.711 & 0.067 & {$[0.000-0.401]$} \\
\hline IPQ-R: Illness coherence & $8.06(3.34)$ & $6.80(2.54)$ & 2.378 & 0.019 & 0.427 & {$[0.073-0.781]$} \\
\hline IPQ-R: Emotional representation & $9.34(3.30)$ & $9.80(3.31)$ & -0.768 & 0.444 & 0.138 & {$[0.000-0.477]$} \\
\hline LOT-R: Optimism & $8.58(2.5 \mathrm{I})$ & $7.23(3.54)$ & 2.428 & 0.017 & 0.436 & {$[0.082-0.790]$} \\
\hline CSQ-R: Pain catastrophizing & $11.2(6.12)$ & $12.2(6.95)$ & -0.86 & 0.389 & 0.152 & {$[0.000-0.495]$} \\
\hline SFQ: Surgical fear & $11.6(14.9)$ & $12.8(15.2)$ & -0.455 & 0.650 & 0.082 & {$[0.000-0.420]$} \\
\hline \multicolumn{7}{|l|}{ Postsurgical variables } \\
\hline NRS' (BPI): Pain intensity & $6.07(2.58)$ & $7.21(2.21)$ & 8.490 & 0.004 & 0.527 & {$[0.196-0.878]$} \\
\hline Frequency: Constant & $28(43.8 \%)$ & $34(56.7 \%)$ & 2.067 & 0.151 & 0.129 & {$[-0.063-0.312]$} \\
\hline Rescue analgesics (yes) & 20 (31.3\%) & $30(50.0 \%)$ & 4.524 & 0.033 & 0.191 & {$[-0.001-0.370]$} \\
\hline Analgesia perineural (yes)g & $12(18.8 \%)$ & $25(41.7 \%)$ & 7.768 & 0.005 & 0.250 & {$[0.057-0.415]$} \\
\hline
\end{tabular}

Notes: Continuous variables are presented as mean (standard deviation); categorical variables are presented as $\mathrm{n}$ (\%); $95 \% \mathrm{Cl}$; ${ }^{a}$ Cohen's $d$ for continuous variables and Pearson's phi $(\varphi)$ coefficient for nominal variables; ${ }^{b}$ comorbidities total = number of comorbid health conditions; 'NRS, $0-10$ from BP; ${ }^{d}$ Pain Total Interference Scale, 0-70 from BPI; eother previous chronic pain states not related to the cause of surgery, but nonetheless frequent; 'frequency of pain = constant vs intermittent or brief; ganalgesia perineural vs intravenous or epidural analgesia.

Abbreviations: $\mathrm{Cl}$, confidence interval; BMI, body mass index; NRS, Numerical Rating Scale; BPI, Brief Pain Inventory; HADS, Hospital Anxiety and Depression Scale; IPQ-R, Illness Perception Questionnaire-Revised; LOT-R, Life Orientation Test-Revised; CSQ-R, Coping Strategies Questionnaire-Revised; SFQ, Surgical Fear Questionnaire; THA, total hip arthroplasty; TKA, total knee arthroplasty.

Regarding pain frequency, there were no significant differences between TKA and THA patients. On rescue analgesia provision, and despite the significance of the $p$-value ( $p=0.033$ ), there were also no differences, since the confidence interval associated with effect size contained the zero value. In addition, TKA patients were provided more often with perineural analgesia $(p=0.005, \varphi=0.250)$.

\section{Intercorrelations between baseline variables $(\mathrm{TI})$ and acute postsurgical pain (T2) in THA and TKA (separate analyses)}

Regarding THA, Table 2 reveals that sex $(r=0.37$, $p=0.002)$, presurgical pain intensity $(r=0.44, p<0.001)$, and other chronic pain states $(r=0.39, p=0.002)$ were significantly correlated with acute postsurgical pain. Concerning psychological factors, only the emotional representation of the condition that led to surgery (OA) ( $r=0.33, p=0.007)$ and optimism $(r=-0.26, p=0.04)$ showed a significant correlation. The remaining psychological variables did not present significant correlations with the outcome variable.

With respect to TKA, Table 3 shows that none of the demographic or clinical factors under analysis showed an association with postsurgical pain. Regarding psychological variables, depression $(r=0.37, p=0.004)$, the emotional representation of the condition that led to surgery (OA) $(r=0.34$, 


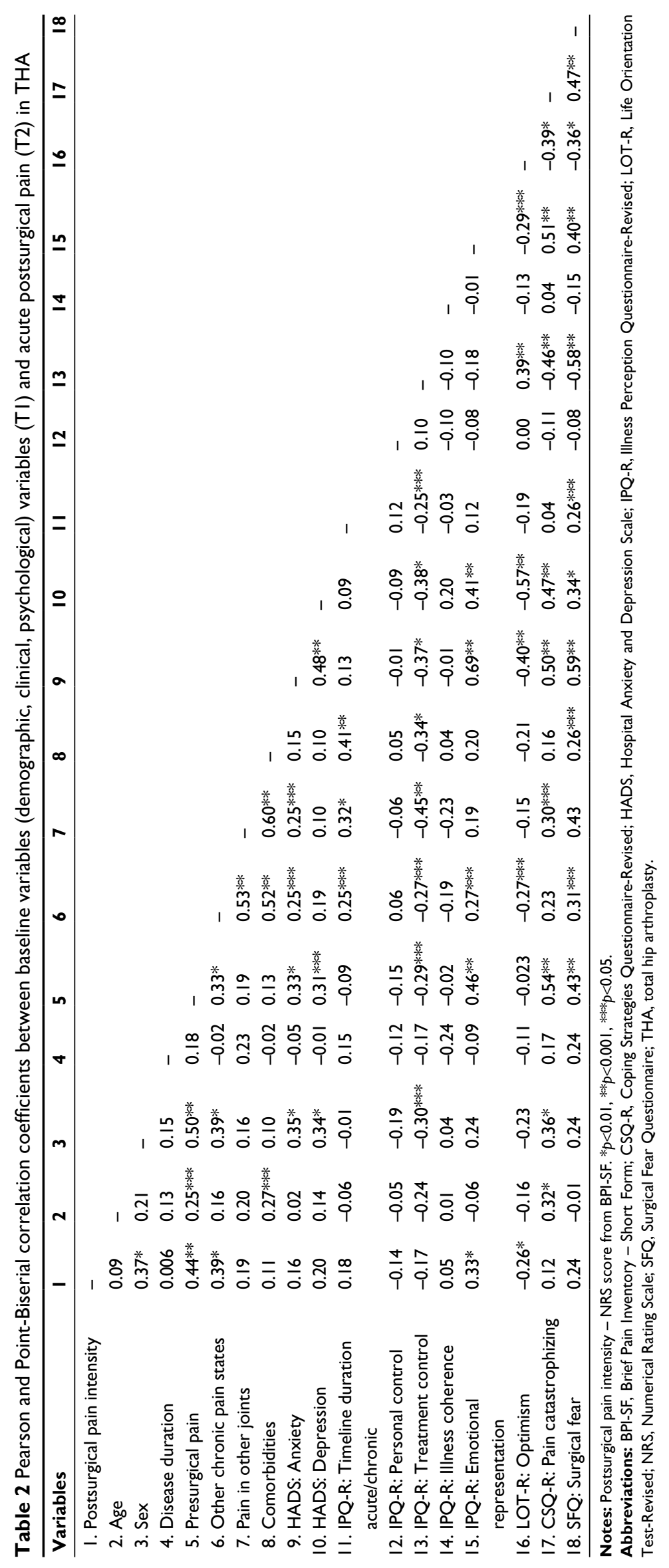




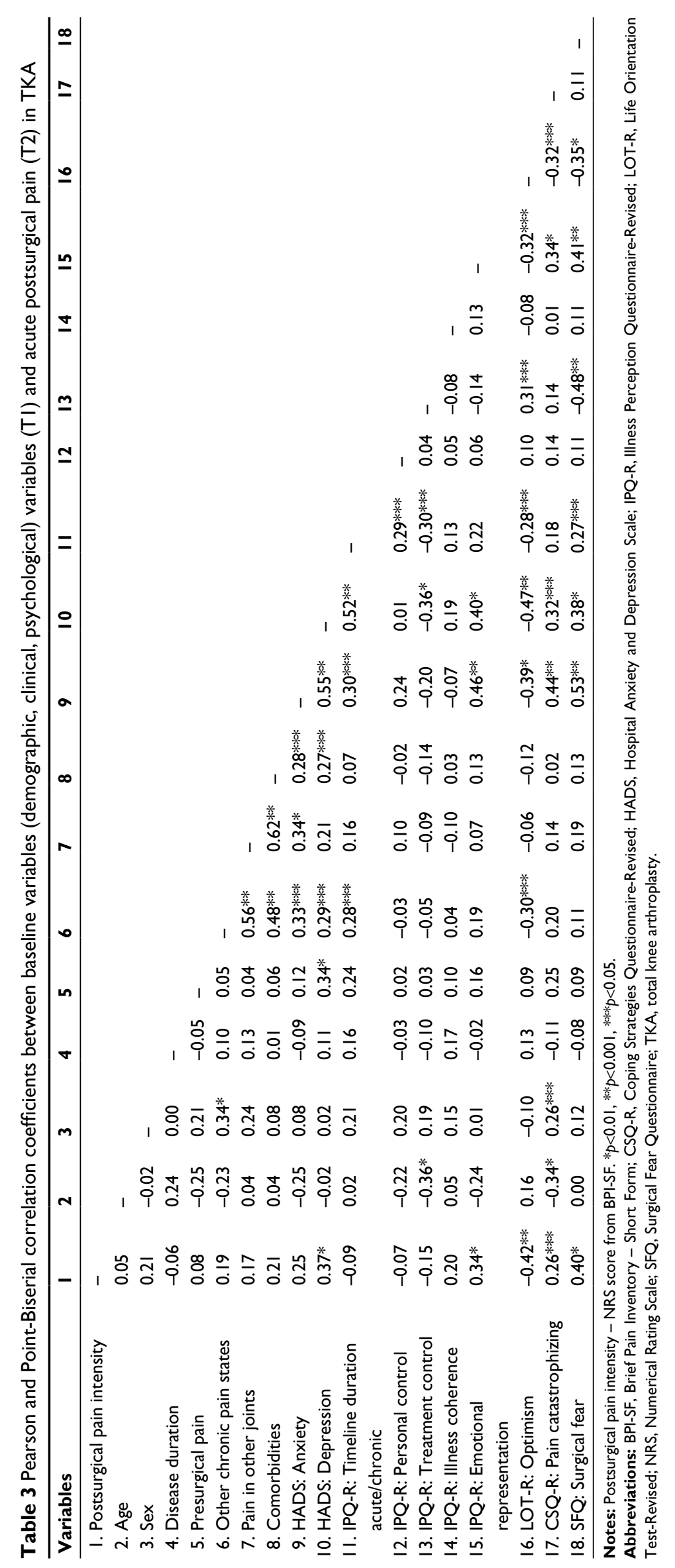


$p=0.007)$, optimism ( $r=-0.42, p=0.001)$, pain catastrophizing $(r=0.26, p=0.048)$, and surgical fear $(r=0.40, p=0.002)$ all correlated significantly with acute postsurgical pain.

\section{Prediction of acute postsurgical pain intensity after THA and TKA}

This study sought to explore whether a common model could predict acute postsurgical pain intensity after each type of arthroplasty. In the regression model under analysis, age and sex (1st block), presurgical pain intensity, and other chronic pain states (2nd block) were considered as potential predictors. Psychological variables that were significantly correlated with the outcome under analysis for both arthroplasty types (emotional representation and optimism) were entered in the last block, which allowed us to investigate the unique role of these variables controlling for demographic and clinical factors.

Table 4 reveals distinct results for each arthroplasty type. For THA, sex showed a predictive role $(t[61]=3.057$, $\beta=-0.371, p=0.003$ ) and explained $14 \%$ of the variance in acute postsurgical pain intensity. Presurgical pain also emerged as a significant predictor $(t[59]=2.344, \beta=0.309$, $p=0.022$ ) and accounted for an additional $13.6 \%$ of the variance. Psychological factors contributed with little additional variance $(0.02 \%)$ for the THA predictive model. In the final THA model, with a total explained variance of $29.4 \%$, presurgical pain was the only variable approaching significance $(t[57]=1.746, \beta=0.254, p=0.086)$.
For TKA (Table 4), neither the variables entered in the first block (sex and age) nor those inserted in the second block (presurgical pain and other chronic pain states) showed significant results in the prediction of acute postsurgical pain, psychological factors being the only significant predictors. In the final model, optimism was the only significant predictor of pain $(t[51]=-2.518, \beta=-0.339, p=0.015)$, and emotional representation $(t[51]=1.895, \beta=0.254, p=0.064)$ presented a trend toward significance, both contributing with additional $20.8 \%$ of the variance. This final model explained $28.1 \%$ of the variance in acute postsurgical pain.

\section{Discussion}

This is the first study aiming to compare acute postsurgical pain intensity and its potential predictors between two types of major joint arthroplasties: THA and TKA. As expected, results showed higher levels of acute pain after TKA comparatively to THA. Moreover, we examined whether the same model could predict acute pain intensity after THA and TKA. Findings revealed different results for THA and TKA. Demographic characteristics and presurgical clinical pain were more relevant predictors for acute postsurgical pain after THA than after TKA. In contrast, presurgical psychological factors were more strongly associated with acute pain after TKA than for THA, namely, optimism emerged as the only and best predictor of postsurgical pain after TKA, over and above demographic and clinical factors. The current study is the first examining these differences with a multivariate

Table 4 Hierarchical multiple regression results for demographic, clinical, and psychological presurgical predictors of acute postsurgical pain intensity 48 hours after THA $(n=64)$ and TKA $(n=60)$

\begin{tabular}{|c|c|c|c|c|c|c|c|c|c|c|}
\hline \multirow[t]{2}{*}{ Variables } & \multicolumn{5}{|c|}{ THA $(n=64)$} & \multicolumn{5}{|c|}{ TKA $(n=60)$} \\
\hline & $t$ & $\beta$ & $R^{2}$ & $\Delta R^{2}$ & $\Delta \boldsymbol{F}$ & $\boldsymbol{t}$ & $\beta$ & $R^{2}$ & $\Delta R^{2}$ & $\Delta \boldsymbol{F}$ \\
\hline Block I & & & & 0.140 & $4.98 I^{*}$ & & & & 0.047 & 1.352 \\
\hline $\operatorname{Sex}^{\mathrm{a}}$ & $3.057^{*}$ & 0.371 & & & & 1.560 & 0.205 & & & \\
\hline $\mathrm{Age}^{\mathrm{b}}$ & 0.125 & 0.015 & & & & 0.537 & 0.071 & & & \\
\hline Block 2 & & & & 0.136 & $5.542 *$ & & & & 0.026 & 0.736 \\
\hline Presurgical pain intensity ${ }^{c}$ & $2.344 * *$ & 0.309 & & & & 0.418 & 0.058 & & & \\
\hline Other chronic pain states ${ }^{d}$ & $1.972 * * *$ & $0.24 I$ & & & & 1.162 & 0.169 & & & \\
\hline Final model & & & 0.294 & & & & & $0.28 I$ & & \\
\hline \multicolumn{11}{|l|}{ Block I } \\
\hline $\operatorname{Sex}^{\mathrm{a}}$ & 0.962 & 0.130 & & & & 1.000 & 0.128 & & & \\
\hline $\mathrm{Age}^{\mathrm{b}}$ & -0.338 & -0.040 & & & & 1.635 & 0.210 & & & \\
\hline \multicolumn{11}{|l|}{ Block 2} \\
\hline Presurgical pain intensityc & $1.746 * * *$ & 0.254 & & & & 0.479 & 0.062 & & & \\
\hline Other chronic pain states ${ }^{d}$ & 1.654 & 0.104 & & & & 0.315 & 0.042 & & & \\
\hline Block 3 & & & & 0.018 & 0.709 & & & & 0.208 & $7.379 *$ \\
\hline Optimism ${ }^{e}$ & -0.760 & -0.092 & & & & $-2.518^{*}$ & -0.339 & & & \\
\hline Emotional representation ${ }^{\mathrm{e}}$ & 0.727 & 0.097 & & & & $1.895^{* * *}$ & 0.254 & & & \\
\hline
\end{tabular}

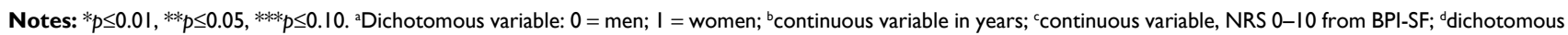
variable: $0=$ no; I = yes; ${ }^{e}$ continuous variable.

Abbreviations: BPI-SF, Brief Pain Inventory-Short Form; NRS, Numerical Rating Scale; THA, total hip arthroplasty; TKA, total knee arthroplasty. 
approach and could prove useful for the design of distinct interventions targeting acute postsurgical pain management, depending on whether the site of arthroplasty is the hip (THA) or the knee (TKA).

\section{Comparing acute postsurgical pain following THA or TKA}

On the question of comparing acute pain levels between THA and TKA patients, this study found that patients undergoing TKA reported more acute pain than those undergoing THA. There is already some empirical knowledge regarding higher acute pain levels after TKA when compared to THA, but the majority of published scientific evidence relates to chronic postsurgical pain rather than to acute postsurgical pain..$^{2-14}$ In fact, regarding chronic postsurgical pain, differences between these two surgeries are well established, with worst results for TKA. ${ }^{3-9}$

Few studies have focused on the comparison of acute pain intensity after THA and TKA. We are aware of studies concerning postarthroplasty acute pain either on $\mathrm{TKA}^{31-40}$ or on THA. ${ }^{28,29}$ Three other studies centered on the comparison between TKA and THA, ${ }^{41,42}$ but did not perform multivariate predictive analyses. We already mentioned the study by Aarons et $\mathrm{al}^{43}$ addressing THA and TKA, taking into account the acute pain period ( 7 days after surgery). They reported a mean pain level of 5.53 (standard deviation $=3.00$ ) for THA and 6.91 (standard deviation $=2.00$ ) for TKA, but did not perform a statistical comparison between these groups. They used small samples (40 THA and 23 TKA patients) and included both primary and revision arthroplasties, which makes the results of this study less robust due to lack of power and heterogeneity of the cases involved. Pang et al's study ${ }^{44}$ did not find differences in acute pain intensity between THA and TKA, but also had a small sample (24 THA vs 26 TKA). Furthermore, they used patient-controlled analgesia (PCA) morphine consumption (number of requests for analgesia and the dose of analgesic used) as a parameter for distinguishing the magnitude of pain intensity between the two major orthopedic surgeries, not directly comparing pain intensity scores as reported by patients. The study by Wylde et al ${ }^{45}$ compared acute pain after THA and TKA, but only assessed pain at rest and was merely descriptive, reporting that $58 \%$ of TKA patients and $47 \%$ of THA patients presented moderate-tosevere pain, with no further comparisons or analyses. Additionally, it presented an asymmetric proportion of patients by surgical group (TKA $=38$ patients vs THA $=67$ patients). Finally, there are two studies focused on the prediction of acute pain after THA and TKA, ${ }^{29,30}$ but in which both THA and TKA are approached jointly, and there is no control of the differential influence of the predictors according to the arthroplasty type.

\section{Predicting acute postsurgical pain following THA or TKA}

A key aim of this study was to investigate whether acute pain after THA and TKA could be predicted by the same model. The results indicate the influence of distinct factors in the explanation of acute pain after THA and TKA. For THA, demographic characters (sex) and presurgical clinical pain had stronger association with acute postsurgical pain than for TKA. Nevertheless, in the final predictive model, sex was no longer significant and presurgical pain only retained marginal significance, thus needing to be revisited in further studies, possibly with larger samples, which would increase power and the likelihood of finding significant effects.

In contrast, presurgical psychological factors were more strongly associated with acute pain after TKA than THA, optimism emerging as the only significant predictor of acute postsurgical pain experience, beyond the effect of demographic and clinical factors. In the multivariate predictive model under analysis, emotional illness representation did retain marginal significance, which merits further exploration in future studies.

Although the reason for the difference between THA and TKA is not clear, it draws attention to the possible complex interplay of the site of arthroplasty and psychological and clinical factors. It is surprising that THA and TKA exhibited a distinct pattern of association between acute pain intensity and demographic, clinical, and psychological variables. Since this is the first study screening acute pain predictors after THA and TKA separately, it is difficult to compare our results to those of other studies.

Nevertheless, our results are in accordance with studies pointing to the more established role of psychological variables on chronic pain after TKA than after THA. ${ }^{9}$ Wylde et al ${ }^{4}$ tested a similar predictive model separately for chronic pain after THA and TKA, but found the same risk factors: depression and pain problems elsewhere. In contrast, Duivenvoorden et a $l^{59}$ suggested the influence of distinct factors for chronic pain resulting from THA and TKA. Specifically, they reported that anxiety had more effect on outcomes after THA and depression influenced the outcomes after TKA, although the outcome variables used in the study included variables other than pain, such as quality of life. 


\section{Limitations of the study}

This is a single site and single country study, which limits its external validity. The sample size per surgery type is also relatively small and the sex composition is unbalanced (more females), which limits generalization of findings.

A potential study bias was that patients could be undergoing three types of analgesic protocols, which were neither controlled in statistical analyses nor empirically standardized, just being recorded a posteriori. Staff within the anesthesiology unit was not fully informed of the study goals in order to assure that normal procedures would be enacted, and thus protocols were tailored to the needs and specificities of each patient, with no attempt made to standardize clinical procedures/protocols. While this reflects the ecological validity of our data, it also might be introducing a potential confounding effect, due to the possible differences among the three protocols in terms of analgesic efficacy. But as we are searching for presurgical predictive regression models, we do not think that this heterogeneity of analgesic regimens prevents samples from being compared with the same presurgical predictive model.

The major strengths of the current study are that: 1) this is the first predictive study examining acute pain after THA and TKA separately, also comparing them; 2) the first study of its kind to assess diverse psychological variables that cover cognitive and emotional factors, while also controlling for demographic and clinical variables; and 3) the multivariate predictive model shows enough robustness, explaining nearly one-third of the explained variance in acute postsurgical pain intensity for both surgical procedures.

\section{Clinical, practical implications, and conclusion}

This study extends prior research and enhances understanding of predictors of acute pain following THA and TKA. Unlike other studies, ${ }^{6,10,30}$ it did not focus on THA and TKA patients jointly. Instead, it compared these two types of surgery and established, through multivariate analyses, distinct predictors of acute postsurgical pain for each surgical major joint procedure. Since distinct predictors were found for THA and TKA, the question arises whether research on acute pain following arthroplasty should combine THA and TKA patients, as has been done in a variety of studies. ${ }^{10,11,29,30}$ The current results support the argument that these two surgeries, at least with regard to acute pain, should be approached separately.

Finally, current findings inform health care professionals about the relevance of implementing distinct interventions concerning acute postarthroplasty pain management. In THA patients, more efforts should be made to control for presurgical pain, either through more aggressive analgesic strategies or by enhancing psychological presurgical pain coping strategies. Instead, in TKA patients, psychological interventions directed at managing psychological factors need to be considered. More specifically, a blend of techniques that would address emotional difficulties, negative cognitions about surgery and pain, and a pessimistic profile seems to be indicated. Cognitive restructuring (cognitive therapy) and hypnosis have both been proven to be efficacious on various pain and surgery relevant outcomes. ${ }^{60-63}$ To deal with more cognitive and personality factors, such as optimism, techniques such as the visualization technique "Best Possible Self" have been successfully employed in promoting optimism ${ }^{64,65}$ and pain reduction. ${ }^{66}$ Presurgical psychological preparation for surgical procedures has already proven to be efficacious. ${ }^{63,67}$ This study's findings also indicate that differential interventions should be designed that take into account the specificity of acute pain predictors for each arthroplasty type.

\section{Acknowledgment}

This work was supported by two grants (SFRH/BD/ 36368/2007 and SFRH/BPD/103529/2014) from the Portuguese Foundation of Science and Technology.

\section{Author contributions}

All authors discussed the results and commented on the manuscript. All authors made substantial contributions to conception and design, acquisition of data, and analysis and interpretation of data. All authors contributed toward data analysis, drafting and critically revising the paper and agree to be accountable for all aspects of the work.

\section{Disclosure}

We declare that none of the authors have any financial or other relationships that might lead to conflict of interest. The authors report no conflicts of interest in this work.

\section{References}

1. Kurtz S, Ong K, Lau E, Mowat F, Halpern M. Projections of primary and revision hip and knee arthroplasty in the United States from 2005 to 2030. J Bone Joint Surg Am. 2007;89(4):780-785.

2. Brander VA, Stulberg SD, Adams AD, et al. Predicting total knee replacement pain - a prospective, observational study. Clin Orthop Relat Res. 2003;416:27-36.

3. Bachmeier CJ, March LM, Cross MJ, et al. A comparison of outcomes in osteoarthritis patients undergoing total hip and knee replacement surgery. Osteoarthritis Cartilage. 2001;9:137-146. 
4. Wylde V, Hewlett S, Learmonth ID, Dieppe P. Persistent pain after joint replacement: prevalence, sensory qualities, and postoperative determinants. Pain. 2011;152:566-572.

5. Beswick AD, Wylde V, Gooberman-Hill R, Blom A, Dieppe P. What proportion of patients report long-term pain after total hip or knee replacement for osteoarthritis? A systematic review of prospective studies in unselected patients. BMJ Open. 2012;2(1):e000435.

6. Pinto PR, McIntyre T, Ferrero R, Almeida A, Araújo-Soares V. Risk factors for moderate and severe persistent pain in patients undergoing total knee and hip arthroplasty: a prospective predictive study. PLoS One. 2013;8(9):e73917.

7. Pinto PR, McIntyre T, Ferrero R, Araújo-Soares V, Almeida A. Persistent pain after total knee or hip arthroplasty: differential study of prevalence, nature and impact. J Pain Res. 2013;3(6):691-703.

8. Bourne RB, Chesworth B, Davis A, et al. Comparing patient outcomes after THA and TKA: is there a difference? Clin Orthop Relat Res. 2010;468(2):542-546.

9. Vissers MM, Bussmann JB, Verhaar JA, Busschbach JJ, Bierma-Zeinstra S, Reijman M. Psychological factors affecting the outcome of total hip and knee arthroplasty: a systematic review. Semin Arthritis Rheum. 2012;41:576-588.

10. Hamel MB, Toth M, Legedza A, Rosen MP. Joint replacement surgery in elderly patients with severe osteoarthritis of the hip or knee. Arch Intern Med. 2008;168:1430-1440.

11. Ebrahimpour PB, Do HT, Bornstein LJ, Westrich GH. Relationship between demographic variables and preoperative pain and disability in 5945 total joint arthroplasties at a single institution. J Arthroplasty. 2011;26(6):133-137.

12. Singh JA, Lewallen DG. Medical and psychological comorbidity predicts poor pain outcomes after total knee arthroplasty. Rheumatology. 2013;52(5):916-923.

13. Sakellariou VI, Poultsides LA, Ma Y, Bae J, Liu S, Sculco TP. Risk assessment for chronic pain and patient satisfaction after total knee arthroplasty. Orthopedics. 2016;39(1):55-62.

14. Lewis GN, Rice DA, McNair PJ, Kluger M. Predictors of persistent pain after total knee arthroplasty: a systematic review and meta-analysis. $\mathrm{Br}$ J Anaesth. 2015;114(4):551-561.

15. Gordon DB, Leon-Casasola OA, Wu CL, Sluka KA, Brennan TJ, Chou R. Research gaps on practice guidelines for acute postoperative pain management in adults: findings from a review of the evidence for an American Pain Society Clinical Practice Guideline. J Pain. 2016;17:158-166.

16. Macintyre PE, Schug SA, Scott DA, Visser EJ, Walker SM; Working Group of the Australian and New Zealand College of Anaesthetists and Faculty of Pain Medicine. Acute Pain Management: Scientific Evidence, 3rd ed. Melbourne: Australian and New Zealand College of Anaesthetists and Faculty of Pain Medicine; 2010.

17. Schug SA. The global year against acute pain. Anaesth Intens Care. 2011;39:11-14.

18. Chapman CR, Tuckett RP, Song CW. Pain and stress in a systems perspective: reciprocal neural, endocrine, and immune interactions. J Pain. 2008;9:122-145.

19. Charlton JE. Acute and postoperative. In: Charlton E, editor. Core Curriculum for Professional Education in Pain. Seattle: IASP Press; 2005:125-138.

20. Apfelbaum JL, Chen C, Mehta SS, et al. Postoperative pain experience: results from a national survey suggest postoperative pain continues to be undermanaged. Anesth Analg. 2003;97:534-540.

21. Bonnet F, Marret E. Postoperative pain management and outcome after surgery. Best Pract Res Clin Anaesthesiol. 2007;21:99-107.

22. Bruce J, Thornton AJ, Scott NW, et al. Chronic preoperative pain and psychological robustness predict acute postoperative pain outcomes after surgery for breast cancer. Br J Cancer. 2012;107(6):937-946.

23. Ip HY, Abrishami A, Peng PW, et al. Predictors of postoperative pain and analgesic consumption: a qualitative systematic review. Anesthesiology. 2009;111:657-677.
24. Pinto PR, McIntyre T, Almeida A, Araújo-Soares V.The mediating role of pain catastrophizing in the relationship between presurgical anxiety and acute postsurgical pain after hysterectomy. Pain. 2012;153:218-226.

25. Sobol-Kwapinska M, Bąbel P, Plotek W, Stelcer B. Psychological correlates of acute postsurgical pain: a systematic review and meta-analysis. Eur J Pain. 2016;20(10):1573-1586.

26. Gerbershagen HJ, Pogatzki-Zahn E, Aduckathil S, et al. Procedurespecific risk factor analysis for the development of severe postoperative pain. Anesthesiology. 2014;120(5):1237-1245.

27. Khan RS, Skapinakis P, Ahmed K, et al. The association between preoperative pain catastrophizing and postoperative pain intensity in cardiac surgery patients. Pain Med. 2012;13(6):820-827.

28. Özalp G, Sarioglu R, Tuncel G, Aslan K, Kadiogullari N. Preoperative emotional states in patients with breast cancer and postoperative pain Acta Anaesthesiol Scand. 2003;47(1):26-29.

29. Liu SS, Buvanendran A, Rathmell JP, et al. Predictors for moderate to severe acute postoperative pain after total hip and knee replacement. Int Orthop. 2012;36(11):2261-2267.

30. Pinto PR, McIntyre T, Ferrero R, Almeida A, Araujo-Soares V. Predictors of acute post-surgical pain and anxiety following primary total hip and knee arthroplasty. J Pain. 2013;14(5):502-515.

31. Kornilov N, Lindberg MF, Gay C, et al. Factors related to postoperative pain trajectories following total knee arthroplasty: a longitudinal study of patients admitted to a russian orthopaedic clinic. Pain Res Treat. Epub 2016 June 15. Available from: http://dx.doi.org/10.1155/2016/3710312.

32. Lindberg MF, Miaskowski C, Rustøen T, et al. Preoperative pain, symptoms, and psychological factors related to higher acute pain trajectories during hospitalization for total knee arthroplasty. PLoS One. 2016;11(9):e0161681

33. Lindberg MF, Miaskowski C, Rustøen T, et al. The impact of demographic, clinical, symptom and psychological characteristics on the trajectories of acute postoperative pain after total knee arthroplasty. Pain Med. Epub 2016 May 10. Available from: http://dx.doi.org/10.1093/pm/ pnw080.

34. Lunn TH, Gaarn-Larsen L, Kehlet H. Prediction of postoperative pain by preoperative pain response to heat stimulation in total knee arthroplasty. Pain. 2013;154:1858-1885.

35. Rakel BA, Blodgett NP, Zimmerman MB, et al. Predictors of postoperative movement and resting pain following total knee replacement. Pain. 2012;153(11):2192-2203.

36. Roth ML, Tripp DA, Harrison MH, Sullivan M, Carson P. Demographic and psychosocial predictors of acute perioperative pain for total knee arthroplasty. Pain Res Manag. 2007;12(3):185-194.

37. Thomazeau J, Rouquette A, Martinez V, et al. Acute pain factors predictive of post-operative pain and opioid requirement in multimodal analgesia following knee replacement. Eur J Pain. 2016;20(5): 822-832.

38. Barrington JW, Lovald S, Ong K, et al. Postoperative pain after primary total knee arthroplasty: comparison of local injection analgesic cocktails and the role of demographic and surgical factors. J Arthroplasty. 2016;31(9):288-292.

39. Kim SH, Yoon KB, Yoon DM, Yoo JH, Ahn KR. Influence of centrally mediated symptoms on postoperative pain in osteoarthritis patients undergoing total knee arthroplasty: a prospective observational evaluation. Pain Pract. 2015;15(6):E46-E53.

40. Torres-Claramunt R, Hinarejos P, Amestoy J, et al. Depressed patients feel more pain in the short term after total knee arthroplasty. Knee Surg Sports Traumatol Arthrosc. 2016:1-6.

41. Erlenwein J, Przemeck M, Degenhart A, et al. The influence of chronic pain on postoperative pain and function after hip surgery: a prospective observational cohort study. J Pain. 2016;17(2):236-247.

42. Pagé MG, Katz J, Curtis K, et al. Acute pain trajectories and the persistence of post-surgical pain: a longitudinal study after total hip arthroplasty. J Anesthesia. 2016;30(4):568-577.

43. Aarons H, Hall G, Hughes S, Salmon P. Short-term recovery from hip and knee arthroplasty. J Bone Joint Surg [Br]. 1996;78:555-558. 
44. Pang WW, Hsu TC, Tung CC, Hung CP, Chang DP, Huang MH. Is total knee replacement more painful than total hip replacement? Acta Anaesthesiol Sin. 2000;38(3):143-148.

45. Wylde V, Rookerb J, Halliday L, Blom A. Acute postoperative pain at rest after hip and knee arthroplasty: severity, sensory qualities and impact on sleep. Orthop Traumatol Surg Res. 2011;97:139-144.

46. Cleeland C, Ryan KM. Pain assessment: global use of the brief pain inventory. Ann Acad Med Singapore. 1994;23:129-138.

47. Cronbach LJ. Coefficient alpha and the internal structure of tests. Psychometrika. 1951;16:297-334.

48. Zigmond AS, Snaith RP. The hospital anxiety and depression scale. Acta Psychiatr Scand. 1983;67:361-370.

49. Moss-Morris R, Weinman J, Petrie KJ, Horne R, Cameron LD, Buick D. The Revised Illness Perception Questionnaire (IPQ-R). Psychol Health. 2002;17:1-16.

50. Scheier MF, Carver CS, Bridges MW. Distinguishing optimism from neuroticism (and trait anxiety, self-mastery, and self-esteem): a reevaluation of the life orientation test. J Pers Soc Psychol. 1994;67(6):1063-1078.

51. Theunissen M, Peters ML, Schouten EG, et al. Validation of the surgical fear questionnaire in adult patients waiting for elective surgery. PLoS One. 2014;9(6):e100225.

52. Riley JL, Robinson ME. CSQ: five factors or fiction? Clin J Pain 1997; 13:156-162.

53. Arbuckle JL. IBM SPSS Amos 22 User's Guide. Crawfordville, FL: Amos Development Corporation; 2013.

54. Jensen MP, Hu X, Potts SL, Gould EM. Single versus composite measures of pain intensity: relative sensitivity for detecting treatment effects. Pain. 2013;154(4):534-538.

55. Spadoni GF, Stratford PW, Solomon PE, Wishart LR. The evaluation of change in pain intensity: a comparison of the $\mathrm{P} 4$ and single-item numeric pain rating scales. J Orthop Sports Phys Ther. 2004;34(4):187-193.
56. Jensen MP, McFarland CA. Increasing the reliability and validity of pain intensity measurement in chronic pain patients. Pain. 1993;55(2):195-203.

57. Kline RB. Principles and Practice of Structural Equation Modeling. New York, NY: Guilford; 2005.

58. Cohen J. Statistical Power Analysis for the Behavioural Sciences. 2nd ed. Hillsdale: Lawrence Erlbaum Associates; 1998.

59. Duivenvoorden T, Vissers M, Verhaar J, et al. Anxiety and depressive symptoms before and after total hip and knee arthroplasty: a prospective multicentre study. Osteoarthritis Cartilage. 2013;21:1824-1840.

60. Jensen MP. A neuropsychological model of pain: research and clinical implications. J Pain. 2010;11(1):2-12.

61. Jensen MP. Psychosocial approaches to pain management: an organizational framework. Pain. 2011;152(4):717-725.

62. Patterson DR, Jensen MP. Hypnosis and clinical pain. Psychol Bull. 2003;129(4):495-521.

63. Tefikow S, Barth J, Maichrowitz S, Beelmann A, Strauss B, Rosendahl J. Efficacy of hypnosis in adults undergoing surgery or medical procedures: a meta-analysis of randomized controlled trials. Clin Psychol Rev. 2013;33(5):623-636.

64. Meevissen Y, Peters M, Alberts H. Become more optimistic by imagining a best possible self: effects of a two week intervention. J Behav Ther Exp Psychiatry. 2011;42(3):371-378.

65. Peters M, Meevissen Y, Hanssen M. Specificity of the best possible self intervention for increasing optimism: comparison with a gratitude intervention. Terapia Psicológica. 2013;31(1):93-100.

66. Hanssen M, Peters M, Vlaeyen J, Meevissen Y, Vancleef L. Optimism lowers pain: evidence of the causal status and underlying mechanisms. Pain. 2013;154:53-58.

67. Johnston M, Vogele C. Benefits of psychological preparation for surgery: a meta-analysis. Ann Behav Med. 1993;15(4):245-256.
Journal of Pain Research

\section{Publish your work in this journal}

The Journal of Pain Research is an international, peer reviewed, open access, online journal that welcomes laboratory and clinical findings in the fields of pain research and the prevention and management of pain. Original research, reviews, symposium reports, hypothesis formation and commentaries are all considered for publication.

\section{Dovepress}

The manuscript management system is completely online and includes a very quick and fair peer-review system, which is all easy to use. Visit http://www.dovepress.com/testimonials.php to read real quotes from published authors. 